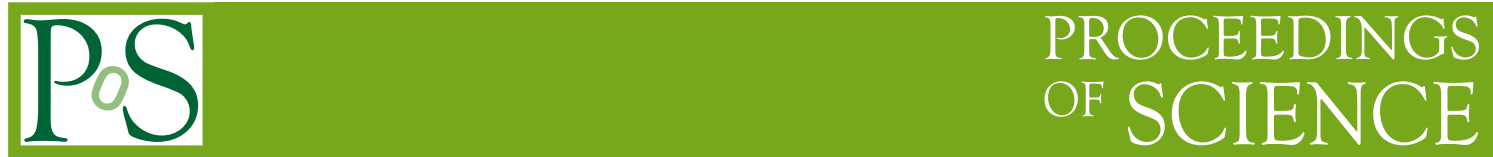

\title{
Dileptons and Photons at RHIC
}

\section{Ismail Zahed*}

Department of Physics and Astronomy

SUNY Stony Brook, Stony Brook NY 11794

E-mail: zahed@tonic.physics.sunysb.edu

Electromagnetic emission in the form of photons or dileptons provide important information on the onset and evolution of a heavy ion collision at ultrarelativistic energies. We briefly summarize the theoretical assessments of the thermalized electromagnetic emissivities from a hot partonic and hadronic medium, and compare them to current experiments at collider energies.

Light Cone 2010 - LC2010

June 14-18, 2010

Valencia, Spain

\footnotetext{
${ }^{*}$ Speaker.
} 


\section{Introduction}

At low and intermediate masses, the electromagnetic emissivities are dominated by thermal emissions. Thermalization at RHIC has been established using both statistical analysis (chemical equilibration) [1] and flow measurements (thermal equilibration) [2]. PHENIX has reported dilepton emissivities all the way to the Dalitz region [3], and more recently extrapolated photon rates [3]. In so far, the electron emissivities in the intermediate mass region have defied most theoretical understanding.

In this talk, I will give a brief overview of our current theoretical understanding of the electromagnetic emissivities by focusing on the photon rates. I will quote the electronic rates. In section 2 , the hadronic photon rates are worked out in terms of the chiral reduction formulae tying the rate to vacuum correlation functions. The hadronic rates overun the partonic rates for a broad range of photon energies. In section 3, we rely on a hydrodynamical evolution to assess the electromagnetic emissivities for 3 distinct experiments to underline the consistency of our approach. Our summary is in section 4.

\section{Hadronic Photon Rates}

For a hadronic gas in thermal equilibrium the number of photon produced per unit four volume and unit three momentum is tied to the electromagnetic current-current correlation function [4]

$$
\frac{q^{0} d N}{d^{3} q}=-\frac{\alpha_{e m}}{4 \pi^{2}} \frac{2}{1+e^{q_{0} / T}} \operatorname{Im} \mathbf{W}^{F}(q)
$$

with $q^{2}=0$ and

$$
\mathbf{W}^{F}(q)=\mathbf{W}_{0}+\int d \pi_{1} \mathbf{W}_{\pi}+\frac{1}{2 !} \int d \pi_{1} d \pi_{2} \mathbf{W}_{\pi \pi}+\cdots
$$

The expansion in $\mathbf{W}^{F}$ is carried over stable hadronic states. At RHIC the baryonic potential is small, so the nucleons can be ignored in the expansion. Here

$$
d \pi_{i}=\frac{d^{3} k_{i}}{(2 \pi)^{3}} \frac{n\left(E_{i}\right)}{2 E_{i}}
$$

counts the pion phase space. We have defined

$$
\begin{aligned}
\mathbf{W}_{0} & =i \int d^{4} x e^{i q \cdot x}\left\langle 0\left|T^{*} \mathbf{J}^{\mu}(x) \mathbf{J}_{\mu}(0)\right| 0\right\rangle \\
\mathbf{W}_{\pi} & =i \int d^{4} x e^{i q \cdot x}\left\langle\pi^{a}\left(k_{1}\right)\left|T^{*} \mathbf{J}^{\mu}(x) \mathbf{J}_{\mu}(0)\right| \pi^{a}\left(k_{1}\right)\right\rangle \\
\mathbf{W}_{\pi \pi} & =i \int d^{4} x e^{i q \cdot x}\left\langle\pi^{a}\left(k_{1}\right) \pi^{b}\left(k_{2}\right)\left|T^{*} \mathbf{J}^{\mu}(x) \mathbf{J}_{\mu}(0)\right| \pi^{a}\left(k_{1}\right) \pi^{b}\left(k_{2}\right)\right\rangle
\end{aligned}
$$

with the sum over isospin subsumed. 
The first contribution in (2.4) is dominated by the transverse part of the isovector correlator and is entirely fixed experimentally by the measured electroproduction data. It vanishes for real photons, i.e. $\mathbf{W}_{0}=0$. The next two terms, $\mathbf{W}_{\pi}$ and $\mathbf{W}_{\pi \pi}$, can be reduced to measurable vacuum correlators by the the chiral reduction formulae [5]. For instance [4, 5]

$$
\begin{aligned}
\mathbf{W}_{\pi}^{F}(q, k) & =\frac{12}{f_{\pi}^{2}} q^{2} \operatorname{Im} \Pi_{V}\left(q^{2}\right) \\
& -\frac{6}{f_{\pi}^{2}}(k+q)^{2} \operatorname{Im} \Pi_{A}\left((k+q)^{2}\right)+(q \rightarrow-q) \\
& +\frac{8}{f_{\pi}^{2}}\left((k \cdot q)^{2}-m_{\pi}^{2} q^{2}\right) \operatorname{Im} \Pi_{V}\left(q^{2}\right) \times \operatorname{Re} \Delta_{R}(k+q)+(q \rightarrow-q)
\end{aligned}
$$

where $\operatorname{Re} \Delta_{R}$ is the real part of the retarded pion propagator, and $\Pi_{V}$ and $\Pi_{A}$ are the transverse parts of the VV and AA correlators. Their spectral functions are related to both $e^{+} e^{-}$annihilation and $\tau$-decay data. The two-pion reduced contribution $\mathbf{W}_{\pi \tau}$ is more involved $[4,6]$.

The dielectron rates follow exactly the same analysis since they correspond to virtual photon emissivities corrected by leptonic matrix elements. Specifically, for $q^{2}<0$,

$$
\frac{d R}{d^{4} q}=\frac{-\alpha^{2}}{3 \pi^{3} q^{2}}\left(1+\frac{2 m_{l}^{2}}{q^{2}}\right)\left(1-\frac{4 m_{l}^{2}}{q^{2}}\right)^{1 / 2} \frac{1}{1+e^{q^{0} / T}} \operatorname{Im}^{F}(q)
$$

Thus

$$
\frac{d R}{d^{4} q}=\frac{2 \alpha}{3 \pi q^{2}}\left(1+\frac{2 m_{l}^{2}}{q^{2}}\right)\left(1-\frac{4 m_{l}^{2}}{q^{2}}\right)^{1 / 2}\left(\frac{q^{0} d N^{*}}{d^{3} q}\right)
$$

which ties the dielectron rate to the virtual photon rate $N^{*}$ again for spacelike momenta. The chiral reduction approach to both dielectrons and photons preserve the nature of this relation. This is not the case for most approaches based on specific hadronic processes. This point is important while analyzing both the electron and photon data reported by PHENIX, since the latter are extrapolated from the former.

In Fig. 1 we compare our hadronic rates with the complete leading order quark-gluon plasma rates [7] for a broad range of photon energies at the highest hadronic temperature $T=190 \mathrm{MeV}$ and for a substantial coupling $\alpha_{s}=3 / 4$. We note that our full hadronic rates are consistently higher than the leading order QGP rates even at the highest photon energies. The hadronic bremsstralung at low photon energies dwarf the near-collinear bremsstralung from the quarks and antiquarks in the QGP [8].

\section{Spectra in Ultra-relativistic Heavy-Ion Collisions}

The hadronic and partonic rates are usually convoluted with the time-evolution history of the fireball from inception at about $1 / 2 \mathrm{fm} / \mathrm{c}$ to thermal freezeout at about 10 to $15 \mathrm{fm} / \mathrm{c}$, depending on the collider energy. The final evolved rates are then folded with the detector acceptance to compare 


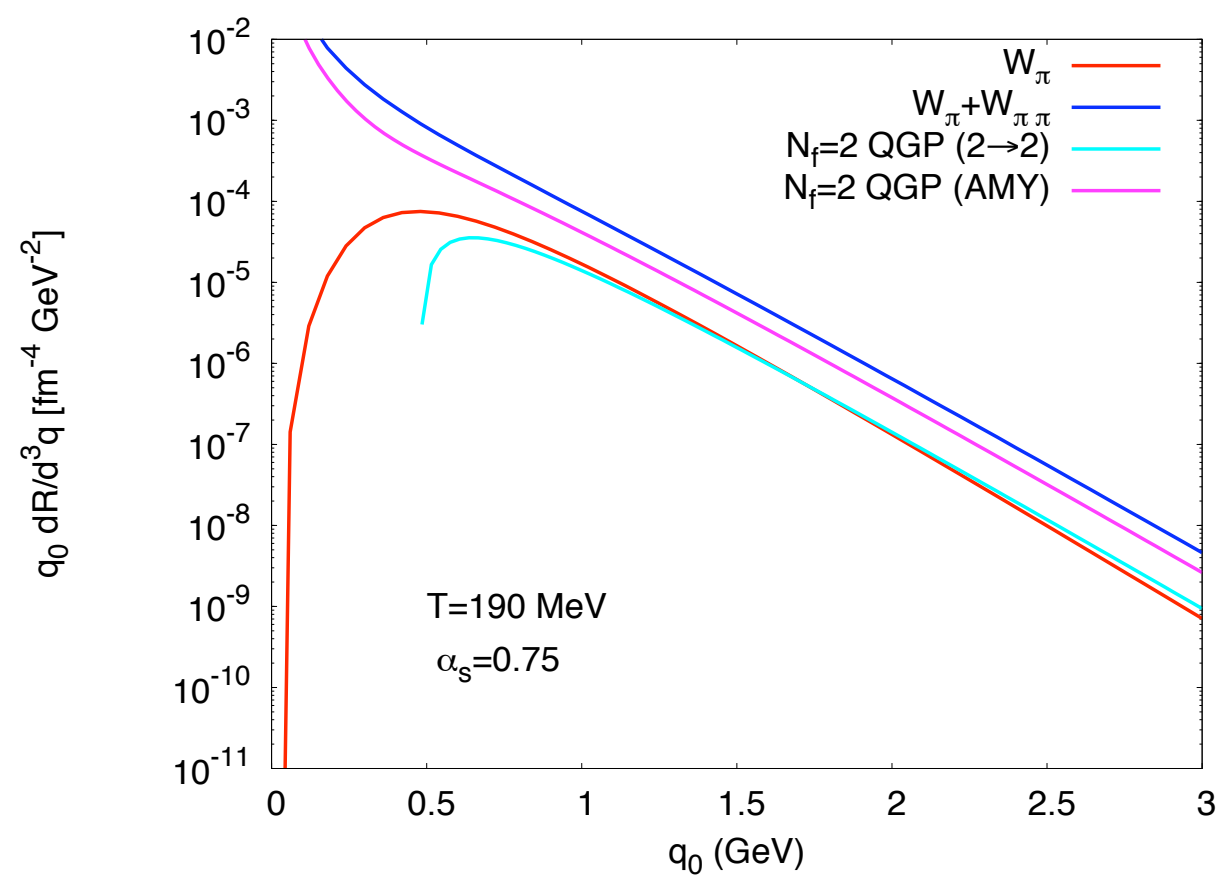

Figure 1: Hadronic photon rates versus partonic rates.

with experiments. In table 1 we summarize our initial parameters based on hydrodynamical evolution as in [8]. The equation of state used for the SPS evolution is based on the bag model, while the equation of state used for both RHIC and LHC is based on lattice results. The SPS evolution ignores baryons in line with the hadronic expansion above. This approximation fares poorly at the SPS, so our emissivity quotes should be viewed as lower bounds.

\begin{tabular}{lcccc}
\hline Parameter & SPS & RHIC 1 & RHIC 2 & LHC \\
\hline \hline$\sqrt{s_{N N}}[\mathrm{~A}-\mathrm{GeV}]$ & 17.3 & 200 & 200 & 5500 \\
$\mathrm{~A}$ & 208 & 197 & 197 & 208 \\
$\sigma_{N N}^{\text {in }}[\mathrm{mb}]$ & 33 & 40 & 40 & 60 \\
$C_{S}$ & 8.06 & 20.8 & 20.8 & 42 \\
$C_{B}$ & 0.191 & 0. & 0. & 0. \\
\hline Centrality: & $0-10 \%$ & $0-20 \%$ & $0-20 \%$ & $0-20 \%$ \\
$\mathrm{~b}[$ fm] & 3 & 4.5 & 4.5 & 4.8 \\
$N_{\text {part }}$ & 340 & 269 & 269 & 293 \\
$\tau_{0}[\mathrm{fm} / \mathrm{c}]$ & 1 & 1 & 0.5 & 0.5 \\
$T\left(\mathbf{r}_{\perp}=0, \tau_{0}\right)[\mathrm{MeV}]$ & 245 & 336 & 398 & 501 \\
$T_{\text {frzout }}[\mathrm{MeV}]$ & 120 & 140 & 160 & 140 \\
\hline
\end{tabular}

Table 1: Hydrodynamical parameters for: SPS, RHIC and LHC.

\subsection{SPS}

In Fig. 2 (left) we detail our evolved photon emissivities from $W_{\pi}$ and $W_{\pi \pi}$ versus the improved 
and leading order QGP rates for WA98 at an impact parameter of $b=3 \mathrm{fm}$ which involves about 340 participants initially. The hadonic rates are substantially larger than the QGP rates both at low and high photon energies. In Fig. 2 (right) we compare our rates with the data from [9]. Remarkably, our hadronic rates fit the photon data both at very low and very high energy although on the lower side. Since our current rates do not include baryons, this is expected. The inclusion of the latters should improve the fits both at low and high energy. In the intermediate mass region, our rates favor the current upper bounds from the data and is consistent with a previous analysis using $W_{\pi}$ and the baryon contributions [4].
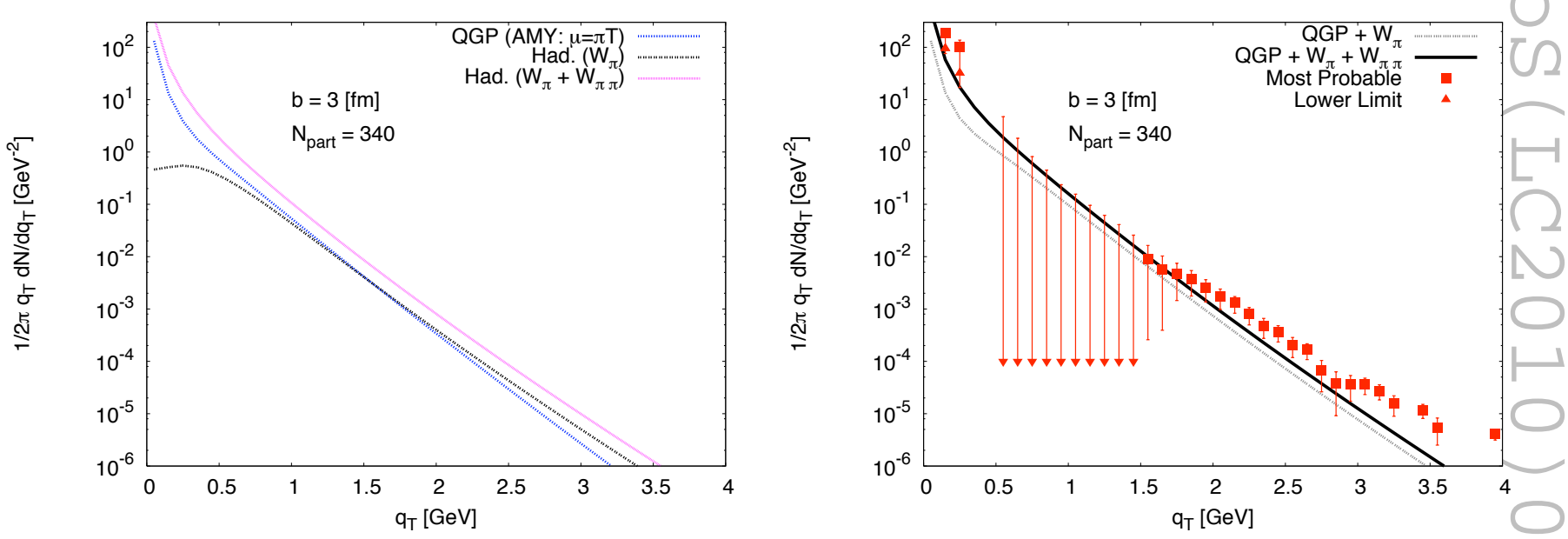

Figure 2: Photon Spectra at the SPS: Rate comparison (left) and Data comparison (right).

\subsection{RHIC}

In Fig. 3 we show the evolved photon rates versus RHIC data for the two different hydrodynamical set ups in Table 1, (left) is RHIC1 and (right) is RHIC2. RHIC2 is a bit more explosive than RHIC1 although both account correctly for the final hadron multiplicities. The scaled pp data by the number of participants are added to account for the contribution from the prompt pp collisions not included in our rate analysis. For RHIC1 our analysis is consistent with the current data as reported above $1 \mathrm{GeV}$. Interestingly enough, for RHIC1 the first two photon points around $1 \mathrm{GeV}$ are well described by the addition of the hadronic rates. The scaled pp emissivities rapidly take over the QGP emissivities around 1.5 GeV to reproduce the data. The photon data appears to favor the parameter set RHIC1 as ooposed to RHIC2. The latter is more explosive with a longer lifetime for the QGP phase than in RHIC1.

\subsection{Photon spectra at LHC}

In Fig. 4 we display our projected photon emissivities at LHC using the hydrodynamical parameter set in Table 1. We expect the conditions at LHC to be a bit more explosive than RHIC, and therefore favoring a parameter choice more in line with RHIC2. The photon emissivities are QGP dominated between $1-2.5 \mathrm{GeV}$ with the hadronic emissivities being comparable to the QGP ones at about $1 \mathrm{GeV}$. The prompt pp photon emissivities take over the yield at about $2.5 \mathrm{GeV}$. 

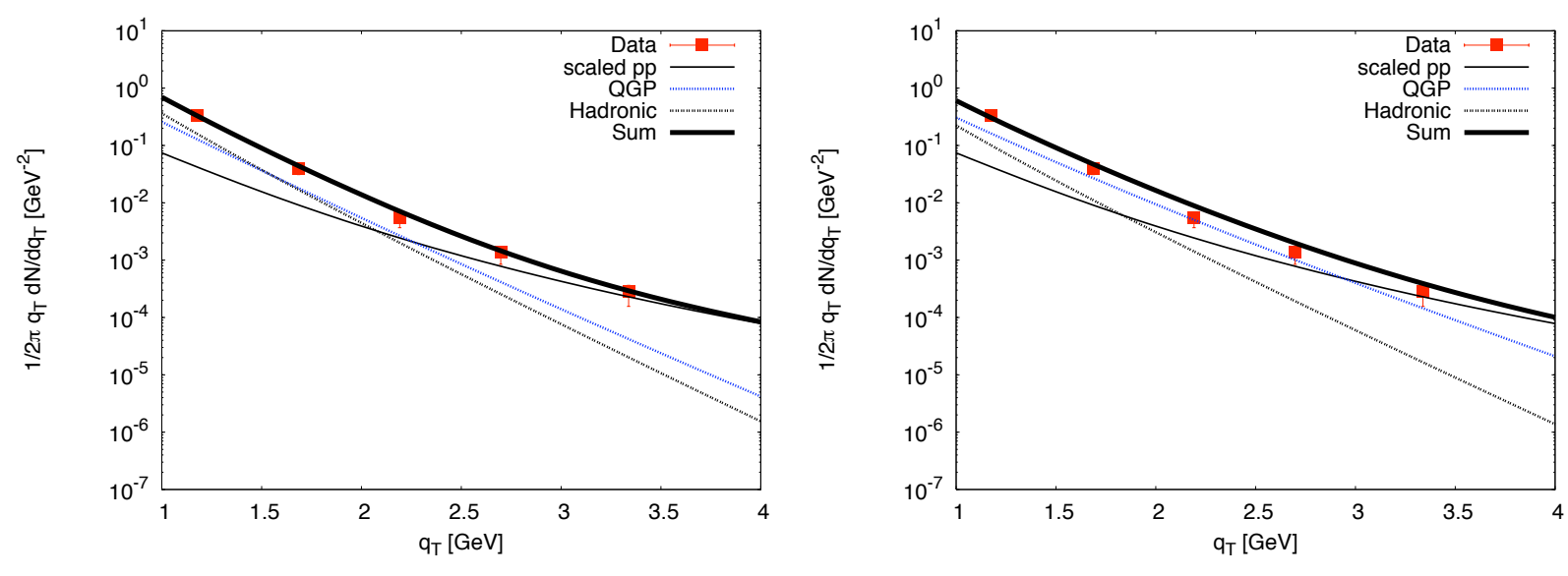

Figure 3: Photon spectra at RHIC versus data: RHIC1 (left) and RHIC2 (right).

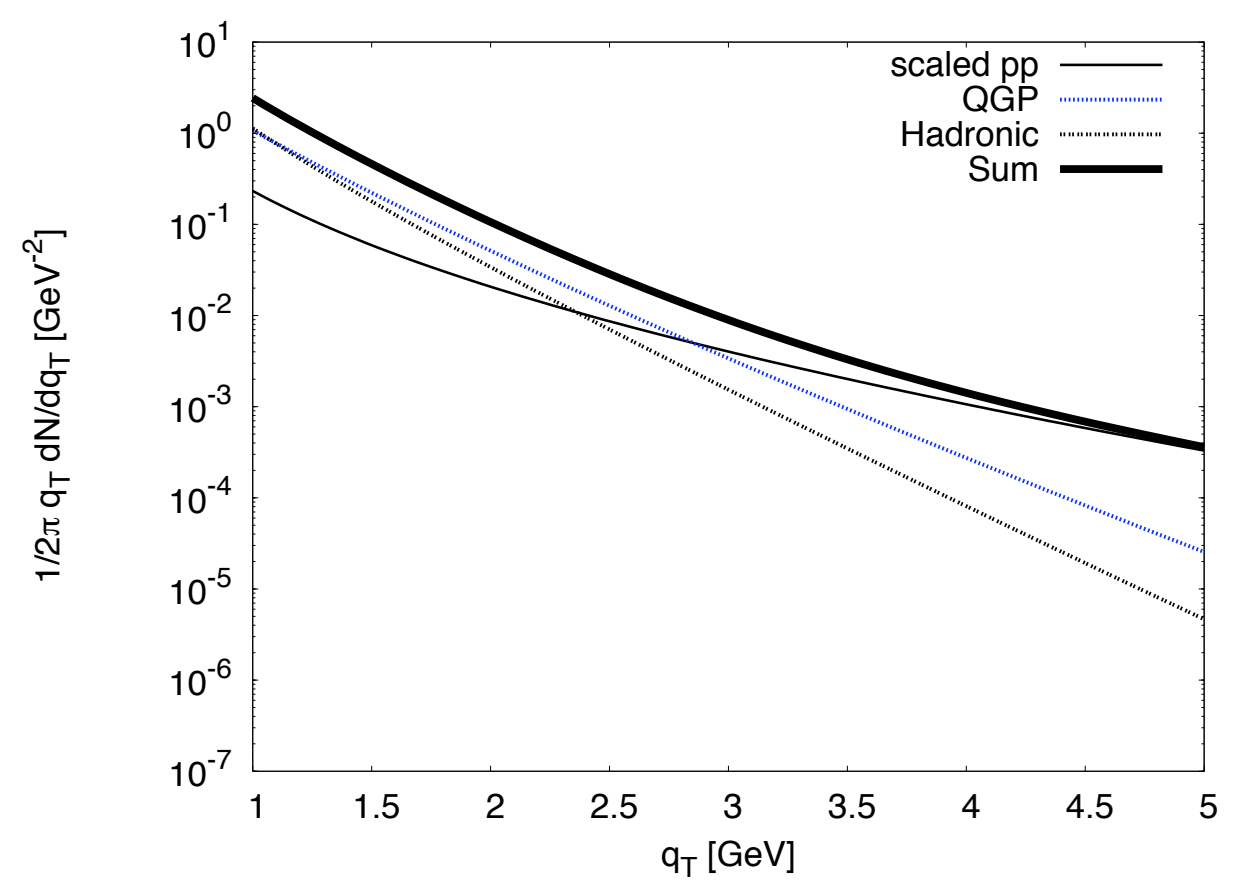

Figure 4: LHC.

\section{Conclusions}

Current theoretical approaches to the electromagnetic emissivities in ultrarelativistic heavy ion colliders are well constrained by partonic/hadronic calculations within a broad range of energies. The exception is the dilepton spectrum reported by PHENIX in the intermediate mass range, where experiment exceeds the best calculations by almost a factor of 1.5. It is worth pointing that the extrapolated photon measurements by PHENIX are consistent with current calculations, making the low mass dielectron discrepancy more puzzling. This said, it is clear that the strength and character of the electromagnetic emissivities point to the formation of a primordial quark and gluon in heavy-ion collisions, with temperatures of the order of $350 \mathrm{MeV}$ at RHIC and perhaps $500 \mathrm{MeV}$ 
at the LHC.

\section{Acknowledgements}

The work presented in this talk was carried in collaboration with Kevin Dusling. This work was supported in part by US DOE grants DE-FG02-88ER40388 and DE-FG03-97ER4014.

\section{References}

[1] M. Kaneta and N. Xu, "Centrality dependence of chemical freezeout in Au-Au collisions at RHIC", [arXiv:nucl-th/045068].

[2] B. Muller and J. L. Nagle, "Results from the relativistic heavy-ion collider", [arXiv: nucl-th/0602029].

[3] A. Drees, "Dileptons and Photons at RHIC energies", Nucl. Phys. A830, 435C (2009).

[4] J. V. Steele, H. Yamagishi and I. Zahed, "Dilepton and photon emission rates from a hadronic gas. II," Phys. Rev. D 56, 5605 (1997) [arXiv:hep-ph/9704414].

[5] H. Yamagishi and I. Zahed, "A master formula for chiral symmetry breaking", Ann. Phys. 247, 292 (1996).

[6] K. Dusling, "Hydrodynamic description of dilepton production", [arXiv:nucl-th/0901.2027]

[7] P. B. Arnold, G. D. Moore and L. G. Yaffe, "Photon emission from quark gluon plasma: Complete leading order results," JHEP 0112, 009 (2001) [arXiv:hep-ph/0111107].

[8] K. Dusling and I. Zahed, "Thermal photons from heavy ion collisions: a spectral function approach", [arXiv:nucl-th/0911.2426].

[9] M. M. Aggarwal et al., "Direct photon production in 158-A-GeV Pb-208+Pb-208 collisions", [arXiv:nucl-ex/0006007]. 